\title{
The Ecological Context of Aerobic Scope in Cost of Transport for Chum Salmon Migrating Upriver
}

\author{
Takaaki K. Abe and Takashi Kitagawa \\ International Coastal Research Center, Atmosphere and Ocean Research Institute, The University of Tokyo 1-19-8, \\ Akahama, Otsuchi, Iwate 028-1102, Japan
}

Keywords: metabolic rate, aerobic scope, cost of transport, thermal adaptation

Ectotherms adjust their thermal performances to various thermal ranges by altering their metabolic rates (Angilletta 2009). Exploring the relationship between thermal niche and the performance of metabolic traits is a controversial topic in the thermal adaptation of ectotherms. Aerobic scope (AS), the difference between resting metabolic rate (RMR) and maximum (aerobic) metabolic rate (MMR), has been proposed as a functional metric for thermal performance (Pörtner and Farrell 2008). The thermal sensitivity of AS is described by the thermal performance curve, where AS increases with temperature up to a peak (which is defined as the optimum temperature of aerobic scope, $T_{\mathrm{optAS}}$ ), and declines thereafter (Payne et al. 2016). Sub-optimal temperatures are known as pejus temperatures $\left(T_{\mathrm{pej}}\right)$. It has been reported that some salmonid species exhibit a variety of thermal profiles for AS curves, and the thermal performance curve of each species or population roughly corresponds to their temperature regime (Lee et al. 2003; Eliason et al. 2011; Clark et al. 2011; Eliason et al. 2013; Raby et al. 2016). Although the intra- or inter-specific adaptation of AS has been shown, however, the degree of correspondence between the thermal performance curves and temperature regimes varies in species or populations. For instance, the peaks of thermal performance curves in some populations do not always correspond with the mode of historically experienced temperatures (Clark et al. 2011; Eliason et al. 2013; Raby et al. 2016; Abe et al. 2019). While the studies of thermal performance curves of AS in Pacific salmonids indicated AS is an ecologically relevant trait, the ecological contexts of AS are not fully understood.

In order to explore the ecological meaning of thermal performance for AS, we used the framework of cost of transport (COT) with aerobic power constraint. This model incorporates the effect of maximum sustained speed (critical swim speed, $U_{\text {crit }}$ ) and the recovery time on COT by assuming the constraint of metabolic power supply (critical power $P_{\text {crit }}$ ) (Martin et al. 2015). The COT model, in other words, enables us to discuss the cost of resting and the time limitation of exercise in high-intensity swimming of salmon, which the existing COT model could not access.

$\operatorname{COT}\left(\mathrm{J} \cdot \mathrm{m}^{-1} \cdot \mathrm{kg}^{-1}\right)$ is generally expressed as the amount of energy consumption per unit distance travelled:

$$
\text { COT }=\frac{P_{\text {travel }}}{U_{\text {swim }}-U_{\text {flow }}},
$$

where, $P_{\text {travel }}$ is total power spent on travel $\left(\mathrm{J} \cdot \mathrm{kg}^{-1} \cdot \mathrm{s}^{-1}\right) . U_{\text {swim }}$ and $U_{\text {flow }}$ are the swim speed of fish $\left(\mathrm{m} \cdot \mathrm{s}^{-1}\right)$ and the flow speed against fish swimming direction $\left(\mathrm{m} \cdot \mathrm{s}^{-1}\right)$, respectively. Thus, $U_{\text {swim }}-U_{\text {flow }}$ is considered as substantial migration speed. COT assumes a U-shaped relationship with $U_{\text {swim, }}$, and then, the optimum swim speed $U_{\text {opt }}$ is estimated from the COT curve as the swim speed, where the $C O T$ was minimized $\left(C O T_{\min }\right)$.

$P_{\text {total }}$ is the sum of the power requirements of maintenance metabolism $P_{\text {metb }}$ and of swimming $P_{\text {swim: }}$ :

$$
P_{\text {total }}=P_{\text {metb }}+P_{\text {swim }} \text {. }
$$

$P_{\text {total }}$ is equal to $P_{\text {travel }}$ in the COT model without aerobic power constraint but not always equal to $P_{\text {travel }}$ in the COT model with the power constraint. In the COT model of the present study, critical power $P_{\text {crit }}$ was assumed, which is defined by the maximum rate of aerobic energy supply and $U_{\text {crit }}$ was also assumed as the swim speed, where $P_{\text {total }}$ is equal to $P_{\text {crit. }}$. Demand for power exceeding $P_{\text {crit }}$ is supplied from anaerobic metabolism, which has a finite capacity. Thus, in the case that fish swim over $P_{\text {crit }}$, the time duration of swimming was limited and the fish needed rest after exhausting anaerobic metabolism.

When fish swim under the intensity of $P_{\text {crit, }}$, the total power requirement, $P_{\text {travel }}$, of migrating fish is equal to $P_{\text {total }}$. Meanwhile, in the case that fish swim over $P_{\text {crit, }}$, the additional costs should be added to $P_{\text {travel }}$. Therefore, the COT model is considered as follows, 


$$
\operatorname{COT}=\left\{\begin{array}{ll}
\frac{P_{\text {total }}}{U_{\text {swim }}-U_{\text {flow }}} & \left(P_{\text {total }} \leq P_{\text {crit }}\right) \\
\frac{P_{\text {total }}+P_{\text {metb }}\left[\frac{y_{\mathrm{r}}\left(P_{\text {total }}-P_{\text {crit }}\right)}{P_{\text {crit }}-P_{\text {metb }}}\right]+\left(y_{\mathrm{r}}-1\right)\left(P_{\text {total }}-P_{\text {crit }}\right)}{U_{\text {swim }}-U_{\text {flow }}} & \left(P_{\text {total }}>P_{\text {crit }}\right)
\end{array},\right.
$$

where, $y_{\mathrm{r}}$ is the recovery yield to restore substrate of anaerobic metabolism. $P_{\text {metb }}\left[y_{\mathrm{r}}\left(P_{\text {total }}-P_{\text {crit }}\right) /\left(P_{\text {crit }}-\right.\right.$ $\left.\left.P_{\text {metb }}\right)\right]$ is the energy expenditure during the resting period and $\left(y_{\mathrm{r}}-1\right)\left(P_{\text {total }}-P_{\text {crit }}\right)$ is the overhead cost to convert a substrate, such as amino acid or lipid, to the substrate of anaerobic metabolism (e.g., glycogen). The parameters in the COT model were used from published respiration data sets (Abe et al. 2019) and estimated parameters (Martin et al. 2015).
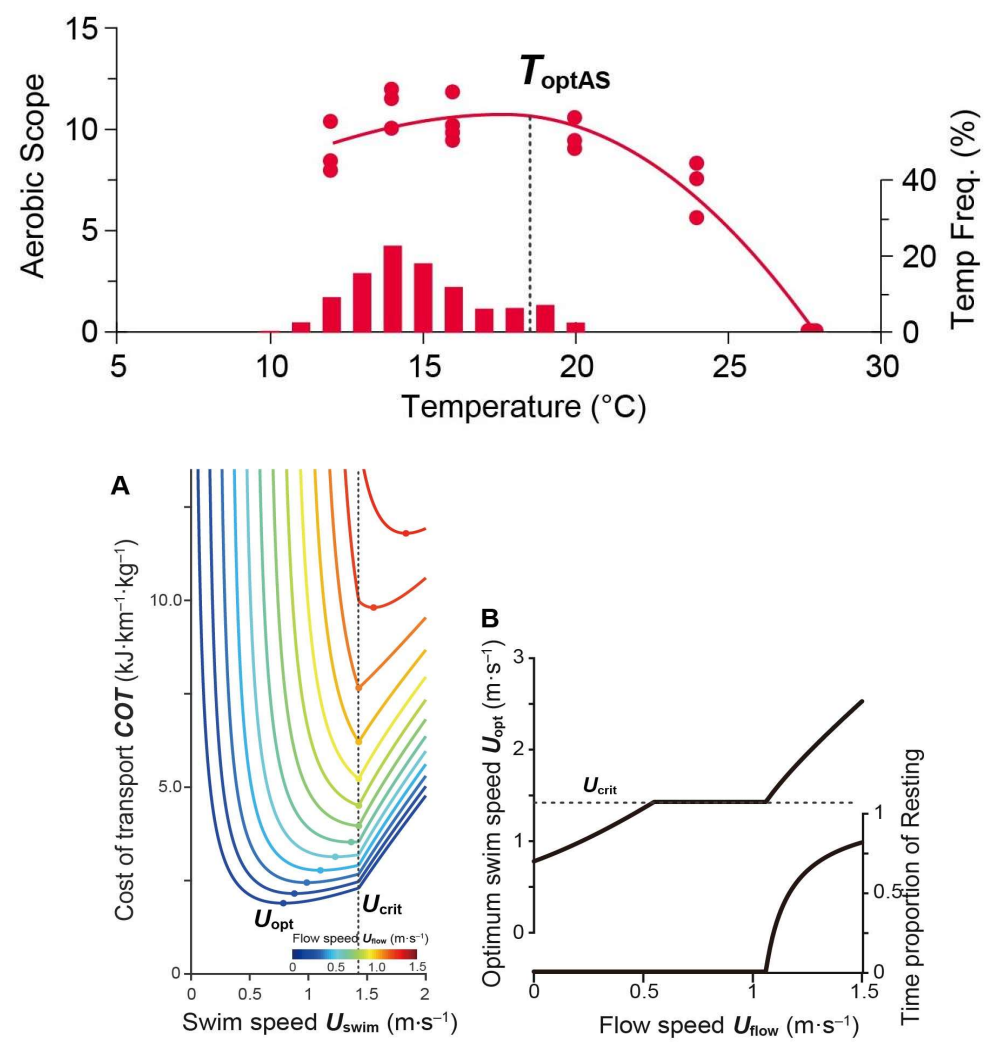

Fig. 1. Aerobic scope of chum salmon in the Kitakami River and environmental temperature. Solid line represents a thermal performance curve fitted for salmon captured in the Kitakami River. The dashed vertical line represents the optimal temperature of AS ( $\left.T_{\text {optAS }}\right)$. The histogram shows the distribution of river temperature from 2013 to 2017 in October, the seasonal timing of chum salmon return in the river.

Fig. 2. Swimming model and simulation and predicted optimal swimming behavior. (A) $C O T$ as a function of the speed the fish swims at $T_{\text {optAAs. }}$ The color indicates flow velocity. Filled circles denote the COT minimizing swim speed at each flow velocity. (B) Estimated $U_{\text {opt }}$ and time proportion of resting under as a function of flow speed.

This COT model was applied to chum salmon (Oncorhynchus keta) migrating upriver in the Kitakami River because their population has a measured performance of AS and the salmon experienced temperatures below $T_{\text {optAS }}$ throughout most of their upriver migration (Abe et al. 2019) (Fig. 1). In the COT model, fish minimize COT by swimming more rapidly through higher flow speed (Fig. 2A). The predicted behavioral response of fish to intermediate flow speed is to cap swimming speed so that power requirements do not exceed $U_{\text {crit, }}$ but under high flow speed, $U_{\text {opt }}$ is higher than $U_{\text {crit }}$ (Fig. 2B). To evaluate the validity of the predicted behavior, we compared the swimming behavior of the chum salmon tagged with accelerometers to the predicted behavior. The swimming behavior of tagged chum salmon altered while migrating upriver (Fig. 3). The fish continuously swam in the lower basin, where flow speed was low, but the fish started to rest occasionally after narrowing, where flow speed starts to increase (Fig. 3B). The fraction of time spent at high swim speed also increased (Fig. 3C).

In order to examine the temperature range for minimizing COT, minimum COT $\left(C O T_{\min }\right)$ was calculated for different temperature and flow speed conditions. In the case of a warmer temperature than $T_{\mathrm{optAS}}, C O T_{\min }$ were higher than $T_{\text {optAS }}$ in any flow speed (Fig. 4A). $C O T_{\min }$ sharply increased at high flow speed and warm temperature further magnified the effect of flow speed (Fig. 4A). In contrast, the simulation in lower temperature than $T_{\text {optAS }}$ suggested the temperature minimizing $C O T_{\min }\left(T_{C O T \min }\right)$ was not always the lowest temperature and $T_{C O T \min }$ depended on flow speed (Fig. 4B). 
The relationship between $C O T_{\min }$ and water temperature for each velocity condition showed that the slower the flow speed condition, the lower $T_{C O T \min }$ (Fig. 5). $T_{C O T \min }$ approached optimal temperature as flow speed increased but did not exceed $T_{\text {optAs }}$ in low flow speeds $\left(<0.9 \mathrm{~m} \cdot \mathrm{s}^{-1}\right)$ (Fig. 5). At high flow speed $\left(\geq 1.0 \mathrm{~m} \cdot \mathrm{s}^{-1}\right)$, however, $T_{C O T \min }$ switched to moving away from $T_{\text {optAs}}$. Interestingly, from intermediate flow speeds $\left(\geq 0.7 \mathrm{~m} \cdot \mathrm{s}^{-1}\right)$, COTmin decreased with the temperature when approaching $T_{\mathrm{optAS}}$, but increased sharply after $T_{\mathrm{optAS}}$. The sharp increase in $C O T_{\min }$ became more remarkable with higher flow speed (Fig. 5). This is because the swim speed minimizing $C O T_{\min }$ becomes higher than $U_{\text {crit }}$ under high flow speed and chum salmon would have to spend a large fraction of its time resting. The simulation suggested that the temperature slightly below $T_{\text {optas }}$ minimized $C O T$ under high flow speed, that is, the temperature range is better to save COT for salmon migrating upriver. In addition, the temperature range minimizing $C O T_{\min }$ corresponded to the empirical temperature of tagged chum salmon migrating upriver (Fig. 5). Our results provided a new perspective on the ecological meaning of aerobic scope. Further quantitative study is needed to better understand the metabolic adaptation of fish to thermal regimes.
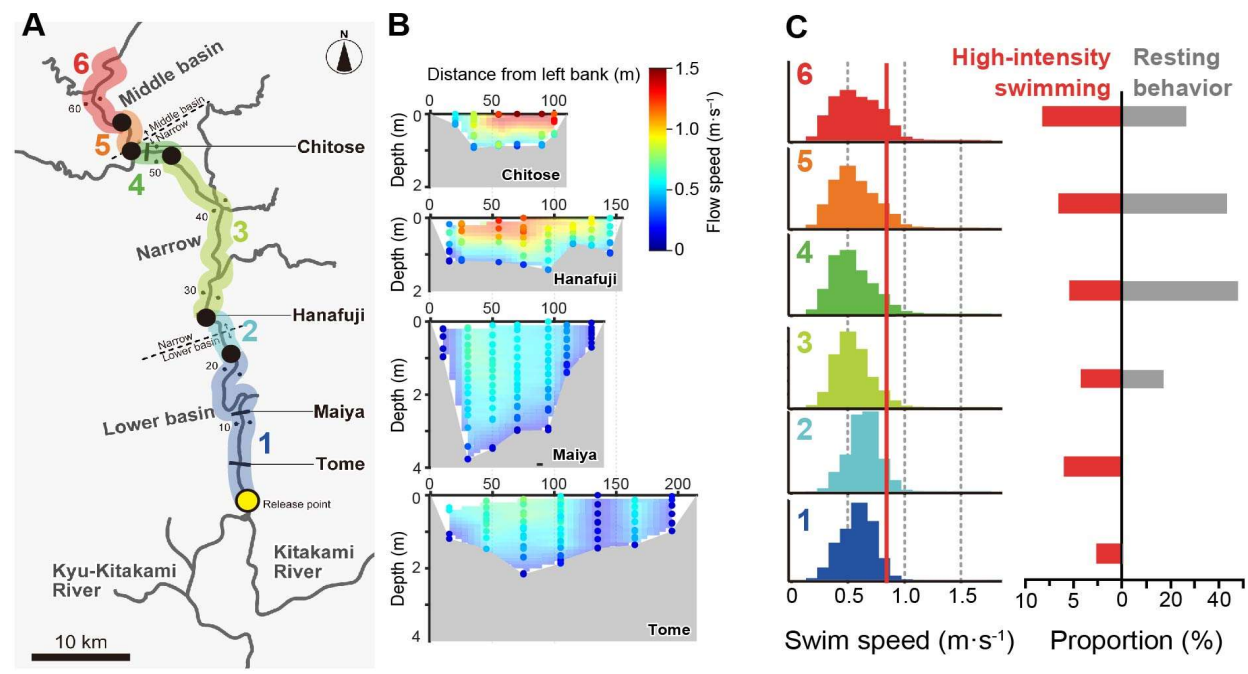

Fig. 3. Study map, the distributions of flow velocity, and the swimming behavior of tagged salmon migrating upriver. (A) Study map of the tracking survey. Filled yellow and black circles represent the release point of tagged fish and the point positioned by VHF tracking, respectively. The time-series data retrieved from the attached accelerometer was classified with six sections divided by the positions. (B) The measurement of flow speed was performed at four bridges (Chitose, Hanafuji, Maiya, and Tome represented in (A)). The colors represent flow velocity and the filled circles indicate the measured values with a flow meter. Colored meshes were created using two-dimensional linear interpolation. (C) Time proportion of time occupied by highintensity swimming inactive phase (red) and time proportion of time occupied by total time (grey) in each section.

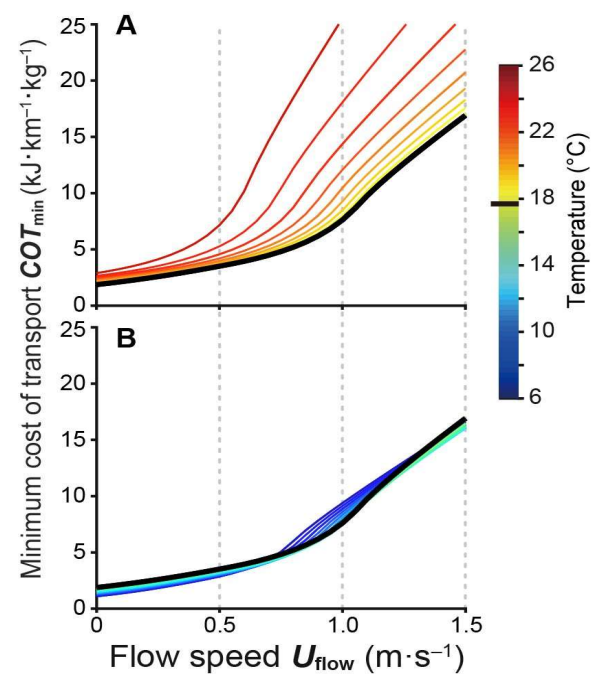

Fig. 4. Minimum $C O T\left(C O T_{\min }\right)$ as a function of flow speed.

The upper (A) and lower (B) figures represent the predictions of $C O T_{\min }$ over and under $T_{\text {optAs }}$, respectively. The colored lines indicate predicted $C O T_{\min }$ in each temperature. The black lines denote predicted $C O T_{\min }$ at $T_{\mathrm{optAS}}$. 


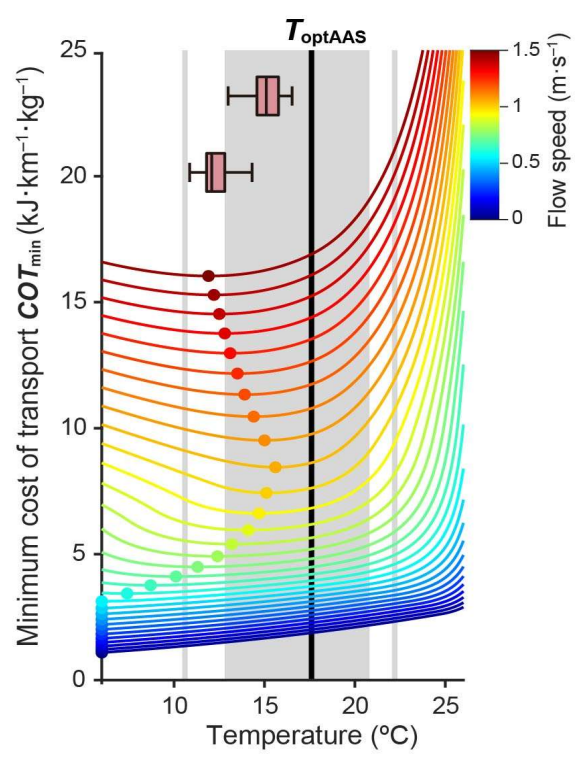

Fig. 5. Minimum $\operatorname{COT}\left(C O T_{\min }\right)$ as a function of temperature. Colored lines indicate predicted $C O T_{\min }$ in each flow speed. Filled circles denote the $C O T_{\min }$ minimizing temperature $\left(T_{\mathrm{COTmin}}\right)$ at each flow speed. The vertical black line represents $T_{\text {optAs}}$. The shaded area indicates the $T_{\text {pej }}$ range (sub-optimal temperature range). The grey lines indicate the temperatures at which AS becomes $80 \%$ of its maximum value. Box plot denotes the experienced temperatures of tagged salmon.

\section{REFERENCES}

Abe, T.K., T. Kitagawa, Y. Makiguchi, and K. Sato. 2019. Chum salmon migrating upriver adjust to environmental temperatures through metabolic compensation. J. Exp. Biol. 222: jeb186189. doi:10.1242/jeb.186189.

Angilletta, M.J. 2009. Thermal adaptation: A theoretical and empirical synthesis. Oxford Univ. Press, New York. $306 \mathrm{pp}$.

Clark, T.D., K.M. Jeffries, S.G. Hinch, and A.P. Farrell. 2011. Exceptional aerobic scope and cardiovascular performance of pink salmon (Oncorhynchus gorbuscha) may underlie resilience in a warming climate. J. Exp. Biol. 214(18): 3074-3081. doi:10.1242/jeb.060517.

Eliason, E.J., T.D. Clark, M.J. Hague, L.M. Hanson, Z.S. Gallagher, K.M. Jeffries, M.K. Gale, D.A. Patterson, S.G. Hinch, and A.P. Farrell. 2011. Differences in thermal tolerance among sockeye salmon populations. Science 332(6025): 109-112. doi: 10.1126/science.

Eliason, E.J., S.M. Wilson, A.P. Farrell, S.J. Cooke, and S.G. Hinch. 2013. Low cardiac and aerobic scope in a coastal population of sockeye salmon Oncorhynchus nerka with a short upriver migration. J. Fish Biol. 82(6): 2104-2112. doi: $10.1111 / \mathrm{jfb} .12120$.

Lee, C.G., A.P. Farrell, A. Lotto, M.J. MacNutt, S.G. Hinch, and M.C. Healey. 2003. The effect of temperature on swimming performance and oxygen consumption in adult sockeye (Oncorhynchus nerka) and coho $(O$. kisutch) salmon stocks. J. Exp. Biol. 206(18): 3239-3251. doi: 10.1242/jeb.00547.

Martin, B.T., R.M. Nisbet, A. Pike, C.J. Michel, and E.M. Danner. 2015. Sport science for salmon and other species: Ecological consequences of metabolic power constraints. Ecol. Lett. 18(6): 535-544. doi:10.1111/ele.12433.

Payne, N.L., J.A. Smith, D.E. van der Meulen, M.D. Taylor, Y.Y. Watanabe, A. Takahashi, T.A. Marzullo, C.A. Gray, G. Cadiou, and I.M. Suthers. 2016. Temperature dependence of fish performance in the wild: Links with species biogeography and physiological thermal tolerance. Funct. Ecol. 30(6): 903-912. doi:10.1111/1365-2435.12618.

Pörtner, H.O., and A.P. Farrell. 2008. Physiology and climate change. Science 322(5902): 690-692. doi:10.1126/science.1163156.

Raby, G.D., M.T. Casselman, S.J. Cooke, S.G. Hinch, A.P. Farrell, and T.D. Clark. 2016. Aerobic scope increases throughout an ecologically relevant temperature range in coho salmon. J. Exp. Biol. 219(12): 1922-1931. doi:10.1242/jeb.137166. 